\title{
Cosmic-Ray induced diffuse emissions from the Milky Way and Local Group galaxies
}

\author{
Troy A. Porter \\ Hansen Experimental Physics Laboratory \\ and \\ Kavli Institute for Particle Astrophysics and Cosmology \\ Stanford University, Stanford, USA \\ email: tporter@stanford.edu
}

\begin{abstract}
Cosmic rays fill up the entire volume of galaxies, providing an important source of heating and ionisation of the interstellar medium, and may play a significant role in the regulation of star formation and galactic evolution. Diffuse emissions from radio to high-energy $\gamma$-rays $(>100 \mathrm{MeV})$ arising from various interactions between cosmic rays and the interstellar medium, interstellar radiation field, and magnetic field, are currently the best way to trace the intensities and spectra of cosmic rays in the Milky Way and other galaxies. In this contribution, I describe our recent work to model the full spectral energy distribution of galaxies like the Milky Way from radio to $\gamma$-ray energies. The application to other galaxies, in particular the Magellanic Clouds and M31 that are now resolved in high-energy $\gamma$-rays by the Fermi-LAT, is also discussed.
\end{abstract}

Keywords. radiation mechanisms: general, radiation mechanisms: nonthermal, (ISM:) cosmic rays, ISM: magnetic fields, galaxies: ISM, (galaxies:) Local Group, (galaxies:) Magellanic Clouds, gamma rays: observations, infrared: galaxies, radio continuum: galaxies

\section{Introduction}

The luminosity of a star-forming galaxy like the Milky Way (MW) is dominated by the relatively narrow frequency range of the spectral energy distribution (SED) from the ultraviolet (UV) to far infrared (FIR), which is due to stellar emission and dust reprocessing in the interstellar medium (ISM). Related to the birth and death of massive stars, cosmic rays (CRs) are pervasive throughout the ISM (see, e.g., Strong et al. 2007 for a recent review). The diffuse emissions arising from various interactions between CRs and the ISM, interstellar radiation field (ISRF - the UV-FIR component of the galactic $\mathrm{SED}$ ), and magnetic field span radio frequencies to high-energy $\gamma$-rays $(>100 \mathrm{MeV})$, but at a lower level of intensity compared to the stellar and dust component. These broadband emissions are currently the best way to trace CR intensities and spectra throughout the MW and other galaxies. Gamma rays are particularly useful in this respect because this energy range gives access to the dominant hadronic component in CRs via the observation of $\pi^{0}$-decay radiation produced by CR nuclei inelastically colliding with the interstellar gas. Understanding the global energy budget of processes related to the injection and propagation of CRs, and how the energy is distributed across the electromagnetic spectrum, is essential to interpret the radio/far-infrared relation (Helou et al. 1985, Murphy et al. 2006), galactic calorimetry (e.g., Völk 1989), and predictions of extragalactic backgrounds (e.g., Thompson et al. 2007, Murphy et al. 2008), and for many other studies. 


\section{Broadband Spectral Energy Distribution for the Milky Way}

The MW is the best studied non-AGN dominated star-forming galaxy, and the only galaxy that direct measurements of CR intensities and spectra are available. However, because of our position inside, the derivation of global properties is not straightforward and requires detailed models of the spatial distribution of the emission. In recent work by Strong et al. (2010) using the GALPROP (e.g., Strong et al. 2000, Moskalenko et al. 2002; also see http://galprop.stanford.edu) and FRaNKIE (Fast Radiative Numerical Kode for Interstellar Emission - Porter et al. 2008) codes, we have calculated the broadband SED of the MW for the first time. Emission by stars and dust is included, together with the diffuse emissions for different $\mathrm{CR}$ propagation models consistent with local CR data. Figure 1 (left) shows the broadband luminosity spectrum of the Galaxy, including the input luminosity for CRs for a $4 \mathrm{kpc}$ halo using a diffusive-reacceleration CR propagation model. Strong et al. (2010) considered a range of halo sizes $(2-10 \mathrm{kpc})$. For this range, the relative decrease in the injected CR proton and helium luminosities is $\sim 10 \%$. For smaller halo sizes, the CRs escape quicker requiring more injected power to maintain the local CR spectrum. In addition, for larger halo sizes CR sources located at further distances can contribute to the local spectrum, which is the normalisation condition, hence less power is required. Contrasting with the CR nuclei, the injected primary CR electron luminosity increases with $z_{h}$, which is required to counter the increased inverseCompton (IC) energy losses in the halo from the longer escape time.
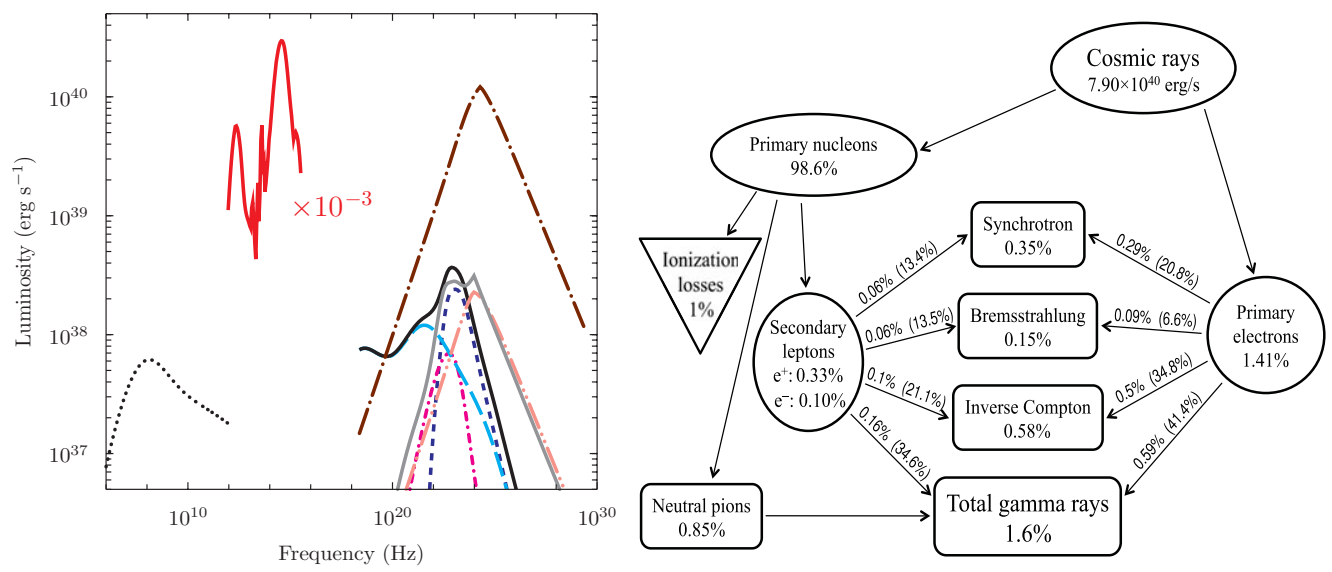

Figure 1. Left: Global CR-induced luminosity spectra of the MW for a diffusive-reacceleration CR propagation model with $z_{h}=10 \mathrm{kpc}$. Line styles: ISRF, including optical and infrared scaled by factor $10^{-3}$ (solid, left side of figure) and components for propagation model - Cosmic rays, protons (long dash dot), helium and heavier nuclei (long dash double dot), primary electrons and secondary electrons and positrons (long dash triple dot); CR-induced diffuse emissions, IC (long dash), bremsstrahlung (medium dash dot), $\pi^{0}$-decay (small dash), total $\gamma$-rays (solid, right side of figure), synchrotron (dotted). Right: Luminosity budget of the MW for the CR propagation model shown in the left panel. The percentage figures are shown with respect to the total injected luminosity in CRs, $7.9 \times 10^{40} \mathrm{erg} \mathrm{s}^{-1}$. The percentages in brackets show the values relative to the luminosity of their respective lepton populations (primary electrons, secondary electrons/positrons).

Figure 1 (right) illustrates the detailed energy budget for the propagation model shown in Fig. 1 (left). The energy channelled into $\gamma$-ray and other secondary production from the CR nuclei component is only a very small fraction of the total power injected in these particles. For the model shown, the total of synchrotron, IC, and bremsstrahlung luminosities for the primary electrons accounts for over half of the total luminosity injected in 
these particles. Note that it is the IC emission at $\gamma$-ray energies that is responsible for the majority of the energy losses, not synchrotron radiation as usually assumed. Including the contribution by secondary electron/positron production, approximately half of the total output in $\gamma$-rays is provided by these particles, even though they count for only $\sim 2 \%$ of the injected power. Non-AGN dominated galaxies like the MW turn out to be very good lepton calorimeters if all the energy-loss processes are taken into account.

\section{Gamma Rays from Local Group Galaxies}

Until recently, the MW was the only galaxy that was resolved in high-energy $\gamma$-rays. However, observations by the Fermi Large Area Telescope (LAT) have extended the sample of resolved star-forming galaxies to include the Magellanic Clouds (Abdo et al. 2010, Abdo et al. 2010) and M31 (Abdo et al. 2010).

Figure 2 (left) shows the background subtracted counts map for a $20^{\circ} \times 20^{\circ}$ region of interest (ROI) surrounding the LMC. The remaining feature is extended emission that is spatially confined to within the LMC boundaries, which are traced by the iso column density contour $N_{\mathrm{H}}=1 \times 10^{21} \mathrm{H} \mathrm{cm}^{-2}$ of neutral hydrogen in the LMC (Kim et al. 2005). The extended $\gamma$-ray emission from the LMC can be resolved into several components. The brightest emission feature is located near $\left(\alpha_{\mathrm{J} 2000}, \delta_{\mathrm{J} 2000}\right) \approx\left(05^{\mathrm{h}} 40^{\mathrm{m}},-69^{\circ} 15^{\prime}\right)$, which is close to the massive star-forming region 30 Doradus (30 Dor) that houses the two Crablike pulsars PSR J0540-6919 and PSR J0537-6910. Excess $\gamma$-ray emission is also seen toward the north and the west of 30 Dor. These bright regions are embedded within a more extended and diffuse glow that covers an area of approximately $5^{\circ} \times 5^{\circ}$.
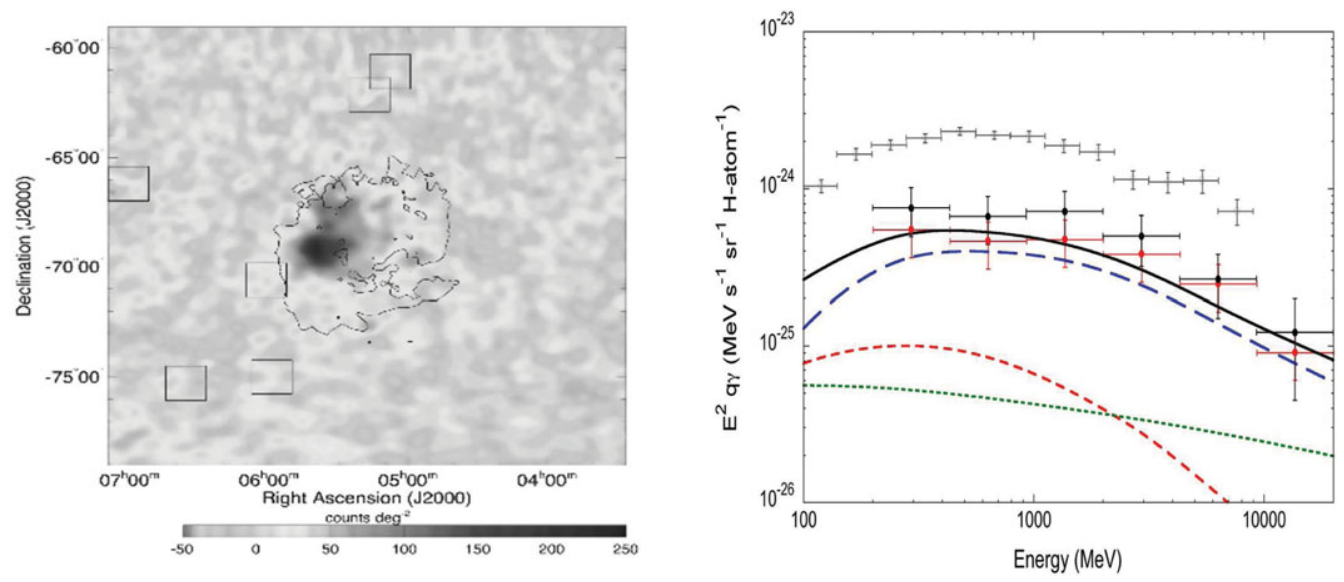

Figure 2. Left: Gaussian kernel $\left(\sigma=0.2^{\circ}\right)$ smoothed count map for the ROI about the LMC after subtraction of the background model for the energy range $200 \mathrm{MeV}-20 \mathrm{GeV}$ and for a pixel size of $0.1^{\circ} \times 0.1^{\circ}$. Overlaid is the $\mathrm{N}(\mathrm{H} \mathrm{I})$ contour of $1 \times 10^{21} \mathrm{H} \mathrm{cm}^{-2}$ of the LMC to indicate the extent and shape of the galaxy. The boxes show the locations of the 6 point sources (background blazars) that were included in the background model. Right: Differential average $\gamma$-ray emissivity spectrum for the LMC. Data for models $\mathcal{H}_{1}$ (upper filled circles) and $\mathcal{H}_{2}$ (lower filled circles ) (see text) and the local ISM emissivity (Abdo et al. 2009 - upper grey data points) are shown. Model lines: solid, predicted total $\gamma$-ray emissivity computed in the framework of a one-zone model for $\mathcal{H}_{2}$; long dashed, $\pi^{0}$-decay; short dashed, bremsstrahlung; dotted, IC. See Abdo et al. 2010 for details.

Most of the gas in the LMC is found in the form of neutral hydrogen and helium with only about $1 \%$ of the total mass ionised. However, the distribution of $\mathrm{H}$ II provides the best fit to the observed $\gamma$-ray emission. It is characterised by a strong emission peak 
near 30 Dor which is attributed to the intense ionising radiation of the massive stars in this highly active region. Even accounting for the 30 Dor emission, the $\mathrm{H}$ II distribution is still a significantly better tracer than the neutral gas of the residual emission. This contrasts with the MW, where the majority of the diffuse $\gamma$-ray emission appears to trace the neutral gas.

Abdo et al. 2010 tested two hypotheses for the origin of the $\gamma$-ray emission from the LMC: $\left(\mathcal{H}_{1}\right)$ all $\gamma$-ray emission from the LMC is attributed to diffuse emission from $\mathrm{CR}$ interactions, and $\left(\mathcal{H}_{2}\right)$ only emission from a disk-like component arises from CR interactions, while the $\gamma$-rays from 30 Dor originate from other sources. The emissivity spectrum derived using these two hypotheses for the LMC is shown in Fig. 2 (right). The integrated emissivity $>100 \mathrm{MeV}$ for the LMC is $\sim 2-4$ times lower than the locally derived emissivity (also shown in the figure). Similar analysis for the SMC (Abdo et al. 2010) and M31 (Abdo et al. 2010) derive emissivities $>100 \mathrm{MeV}$ that are $\sim 6-7$ and $\sim 2$ times lower, respectively. Assuming that the proton-to-electron ratio in these galaxies above a few tens of $\mathrm{GeV}$ is similar to that in the MW and that all of the emission is from diffuse processes, these values point to a general picture where the intensity of the emission is controlled by the power injected by CR sources scaling approximately with the relative star-formation rates in the different systems. Future work combining the $\gamma$ ray, radio, and other data will result in broadband SEDs of these galaxies spanning more than 20 decades in frequency, which can be used to investigate a variety of phenomena related to how energy is injected and cycled within the ISM of galaxies other than the MW.

\section{Acknowledgements}

The Fermi-LAT Collaboration acknowledges support from a number of agencies and institutes for both development and the operation of the LAT as well as scientific data analysis. These include NASA and DOE in the United States, CEA/Irfu and IN2P3/CNRS in France, ASI and INFN in Italy, MEXT, KEK, and JAXA in Japan, and the K. A. Wallenberg Foundation, the Swedish Research Council and the National Space Board in Sweden. Additional support from INAF in Italy and CNES in France for science analysis during the operations phase is also gratefully acknowledged. GALPROP development is supported via NASA Grant Nos. NNX10AE78G and NNX09AC15G.

\section{References}

Abdo, A. A., Ackermann, M., Ajello, M., et al. 2009, ApJ, 703, 1249

Abdo, A. A., Ackermann, M., Ajello, M., et al. 2010, A\&BA, 512, 7

Abdo, A. A., Ackermann, M., Ajello, M., et al. 2010, A\&BA, 523, L2

Abdo, A. A., Ackermann, M., Ajello, M., et al. 2010, A\&BA, 523, 46

Helou, G., Soifer, B. T., \& Rowan-Robinson, M. 1985, ApJL, 298, L7

Kim, S., Staveley-Smith, L., Dopita, M. A., et al. 2005, ApJS, 143, 487

Moskalenko, I. V., Strong, A. W., Ormes, J. F., et al. 2002, ApJ, 565, 280

Murphy, E. J., Helou, G., Braun, R., et al. 2006, ApJ, 638, 157

Murphy, E. J., Helou, G., Kenney, J. D. P., et al. 2008, ApJ, 678, 828

Porter, T. A., Moskalenko, I. V., Strong, A. W., et al. 2008, ApJ, 682, 400

Strong, A. W., Moskalenko, I. V., \& Reimer, O. 2000, ApJ, 537, 763

Strong, A. W., Moskalenko, I. V., \& Ptuskin, V. S. 2007, Ann. Rev. Nuc. Part. Sci. , 57, 285

Strong, A. W., Porter, T. A., Digel, S. W., et al. 2010, ApJL, 722, 58

Thompson, T. A., Quataert, E., \& Waxman, E. 2007, ApJ, 654, 219

Völk, H. J. 1989, A $\mathscr{\mho} A, 218,67$ 


\section{Discussion}

TABATABAEI: Have you tried to derive the XCO conversion factor based on your gammaray data?

PorTER: Concerning the question of whether XCO can be constrained using gammarays, I would say, yes, provided all details of cosmic ray propagation and origin are known. In practice, it is problematic to obtain XCO throughout the Galaxy using gamma-ray data. 